Check for updates

Cite this: RSC Adv., 2018, 8, 153

Received 9th October 2017

Accepted 12th December 2017

DOI: $10.1039 / c 7 r a 11091 j$

rsc.li/rsc-advances

\section{Hemocompatible poly(lactic acid) membranes prepared by immobilizing carboxylated graphene oxide via mussel-inspired method for hemodialysis $\dagger$}

Lankun Ma, (D) abc Lilan Huang, ${ }^{\text {ac }}$ Yuzhong Zhang, *ac Lizhi Zhao, ${ }^{\text {ac }}$ Qingping Xin, (D) Hui $\mathrm{Ye}\left(\mathbb{D}^{\mathrm{ac}}\right.$ and Hong $\mathrm{Li}^{\text {ac }}$

Poly(lactic acid) (PLA) is an environmentally friendly material, but the hydrophobicity and poor hemocompatibility of PLA impede its application as hemodialysis membranes. In this study, aiming to improve the hemocompatibility of PLA membranes, dopamine-g-carboxylated graphene oxide (DA- $g$ $\mathrm{GOCOOH}$ ) was synthesized and then immobilized on PLA membranes via a mussel-inspired adhesion method. The effect of carboxyl content of graphene oxide on hemocompatibility was also investigated. Attenuated total reflectance Fourier transform infrared spectra (ATR-FTIR), X-ray photoelectron spectroscopy (XPS) and scanning electron microscopy (SEM) analysis confirmed that DA-g-GOCOOH was successfully immobilized on the PLA membranes. The significant improvement of hydrophilicity and electronegativity of the PLA membranes effectively alleviated the surface adhesion of platelets, prolonged the recalcification time and reduced the hemolysis ratio to less than $0.3 \%$. Moreover, the DA$g-\mathrm{GOCOOH}$ modified PLA membrane showed excellent dialysis performance, especially for the clearance of middle molecule toxin, which was up to $24 \%$. The DA-g-GOCOOH immobilized layers were relatively stable after incubating in water. The present work demonstrated a potential way to improve the hemocompatibility of PLA membranes for hemodialysis.

\section{Introduction}

Biodegradable polymers have attracted much attention due to environment and sustainability issues. ${ }^{1}$ As a promising biomedical material, poly(lactic acid) (PLA) is biodegradable, bioabsorbable, renewable and extensively investigated..$^{1-3}$ However, PLA is relatively hydrophobic and lacks reactive sidechain groups ${ }^{\mathbf{1}}$ when it is used as a human blood-contacting material. Li et al. improved the hemocompatibility of polypropylene by attaching silver nanoparticles capped with TPGS. ${ }^{4}$

\footnotetext{
${ }^{a}$ State Key Laboratory of Separation Membranes and Membrane Processes, Tianjin Polytechnic University, Tianjin 300387, China

${ }^{b}$ School of Textiles, Tianjin Polytechnic University, Tianjin 300387, China

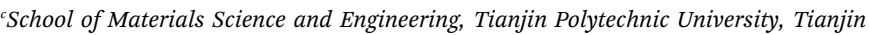
300387, China. E-mail: zhangyz2004cn@vip.163.com

$\uparrow$ Electronic supplementary information (ESI) available: Materials, detailed procedures for preparation of the original PLA membrane, graphene oxide (GO), reduced graphene oxide (rGO), carboxylic graphene oxide (GO-COOH), rGO coating on the PLA membrane surface, FTIR spectra of graphene oxide (GO) and carboxylic graphene oxide (GO-COOH), AFM analysis of carboxylic graphene oxide, XPS and TEM analysis of DA- $g$-GO, photographs and C 1s XPS of rGO, SEM analysis, water contact angle, zeta potential, platelets adhesion, plasma recalcification time analysis, hemolysis ratio of $\mathrm{rGO}$ and DA- $g$-GO modified PLA membranes and the stability of the DA-g-GOCOOH coating layers. See DOI: $10.1039 / \mathrm{c} 7 \mathrm{ra} 11091 \mathrm{j}$
}

Jiang et al., ${ }^{5}$ You et al. ${ }^{6}$ and Gao et al. ${ }^{7}$ grafted heparin onto PE porous membrane surface, PU substrates and PLA membrane surface to develop their hemocompatibility, respectively. Chang et al. grafted zwitterionic poly(sulfobetaine methacrylate) (PSBMA) onto PVDF membrane and PP fibrous membrane via plasma-induced surface copolymerization to improve the materials' blood compatibility. ${ }^{8,9}$ Zhu et al. grafted PSBMA to PLA membrane surface by atom transfer radical polymerization (ATRP) to improve the fouling resistance and hemocompatibility. ${ }^{2}$ Xiong et al. improved the blood compatibility of PLA membrane by bonding heparin via surface cross-linking and glycidyl ether reaction. ${ }^{\mathbf{1 0}} \mathrm{Li}$ et al. immobilized natural hirudin on PLA membrane to develop a hemocompatible PLA membrane through the hydrogen bonding interaction. ${ }^{\mathbf{1 1}}$ However, plasma treatment and ATRP are somewhat complex and may be not suitable for large-scale surface modification. ${ }^{\mathbf{1 2}}$

In recent years, graphene oxide (GO), which contains carboxyl, hydroxy and epoxy functional groups ${ }^{\mathbf{1 3 , 1 4}}$ has attracted many researchers' attention. Much research has been performed to investigate the biocompatibility and toxicity of GO in vivo and in vitro when it is used for biotechnological application. ${ }^{14-17}$ Cheng et al. prepared a biopolymer adhered GO or rGO by a facile biomimetic method and the biopolymer exhibited controllable 2D morphology and excellent biocompatibility. ${ }^{\mathbf{1 8}}$ 
Hu et al. ${ }^{19}$ and Chae et al. ${ }^{20}$ suggested that GO performed good bio-antifouling property on account of its hydrophilicity, negative charge, and surface smoothness.

Huang et al. modified graphene oxide with aptamerconjugated gold nanoparticles and heparin to obtain a targeted anticoagulant. ${ }^{21} \mathrm{He}$ et al. fabricated a hemocompatible and antibacterial polymeric membrane by coating a top layer of graphene oxide and a sulfonated polyanion co-doped hydrogel thin film..$^{22}$ Pinto et al. investigated the biocompatibility of PLA with blending graphene oxide and graphene nanoplatelets respectively. ${ }^{23}$ However, to better understand the hemocompatibility of PLA membrane surface immobilizing GO, further research is urgently needed.

Dopamine (DA), which can be easily deposited on inorganic and organic substrates, has been widely investigated. ${ }^{24-28}$ Furthermore, DA can be grafted onto various materials, and the as-prepared materials exhibit excellent adhesive ability to solid surfaces. ${ }^{12,18,29-31}$ In the present work, DA molecules were grafted onto carboxylic graphene oxide (GOCOOH) nanoplatelets to obtain mussel inspired adhesive nanoplatelets (DA- $g$ GOCOOH) by carbodiimide chemistry method. Subsequently, DA- $g$-GOCOOH were adhered onto the PLA membrane surface via DA-g-GOCOOH's adhesive ability in tris(hydroxymethyl) aminomethane hydrochloride buffer (Tris-buffer, $\mathrm{pH}$ 8.5). The surface chemistry, morphology, charge, water contact angle and the hemocompatibility (platelet adhesion, clotting time and hemolysis ratio) of the PLA membranes after modification were studied. Furthermore, the dialysis performances of the PLA/(DA- $g$-GOCOOH) membranes were investigated. The procedure of preparing PLA/(DA- $g$-GOCOOH) membrane, blood anticoagulation and hemodialysis was shown in Scheme 1. Herein, the present work aims to explore a novel method to improve the hemocompatibility of PLA membranes for hemodialysis.

\section{Experiments}

\subsection{Preparation DA-g-GOCOOH nanosheets}

Briefly, $0.5 \mathrm{~g}$ GOCOOH (dispersing in $75 \mathrm{~mL} \mathrm{H}_{2} \mathrm{O}$ ) was sonicated for $30 \mathrm{~min}$, and then, EDC (0.1917 g), NHS (0.0575 g) and $125 \mathrm{mg}$ DA were added sequentially. Stir the mixture vigorously under $\mathrm{N}_{2}$ protection (to prevent the self-polymerization of dopamine) at $30{ }^{\circ} \mathrm{C}$. The resultant DA- $g$-GOCOOH were washed with DI water after centrifuging for several times to remove impurities and kept in $\mathrm{N}_{2}$ protection. For comparison, DA- $g$-GO was also prepared by the same method.

The chemical composition and morphology of GOCOOH and DA- $g$-GOCOOH were analysed by using Fourier transform infrared spectroscopy (FTIR, Nicolet iS50, Thermo Scientific, America), X-ray photoelectron spectrometer (XPS, K-Alpha, ThermoFisher, America) and transmission electron microscope (TEM, H7650, HITACHI, Japan).

\subsection{DA-g-GOCOOH coating on the PLA membrane surface and characterization of the DA- $g$-GOCOOH coating layer}

Briefly, the prepared PLA membranes were immersed in DA- $g$ GOCOOH solution with different concentrations $0.5 \mathrm{mg} \mathrm{mL}^{-1}$, $1.0 \mathrm{mg} \mathrm{mL}{ }^{-1}, 1.5 \mathrm{mg} \mathrm{mL}^{-1}, 2.0 \mathrm{mg} \mathrm{mL}^{-1}$ ) dissolved in tris(hydroxymethyl)aminomethane hydrochloride buffer $(10 \mathrm{mM}, \mathrm{pH}$ 8.5, containing $\left.5 \mathrm{mM}, \mathrm{Fe}\left(\mathrm{SO}_{4}\right)_{2}\left(\mathrm{NH}_{4}\right)_{2}\right)$ for $24 \mathrm{~h}$. The DA- $g$ GOCOOH was adhered onto the PLA membrane due to its excellent adhesive ability. Then, the membranes coated with DA-g-GOCOOH were washed with deionized water to remove unfirm DA-g-GOCOOH. The resultant DA- $g$-GOCOOH coated PLA membranes were marked by CG-0.5, CG-1.0, CG-1.5, CG2.0. For comparison, DA- $g$-GO immobilized on the PLA membrane surface was also obtained by the same method. PLA membrane was immersed in $2.0 \mathrm{mg} \mathrm{mL}^{-1}$ DA solution (Trisbuffer, $10 \mathrm{mM}, \mathrm{pH}$ 8.5) for $24 \mathrm{~h}$ to obtain PLA/PDA membrane.
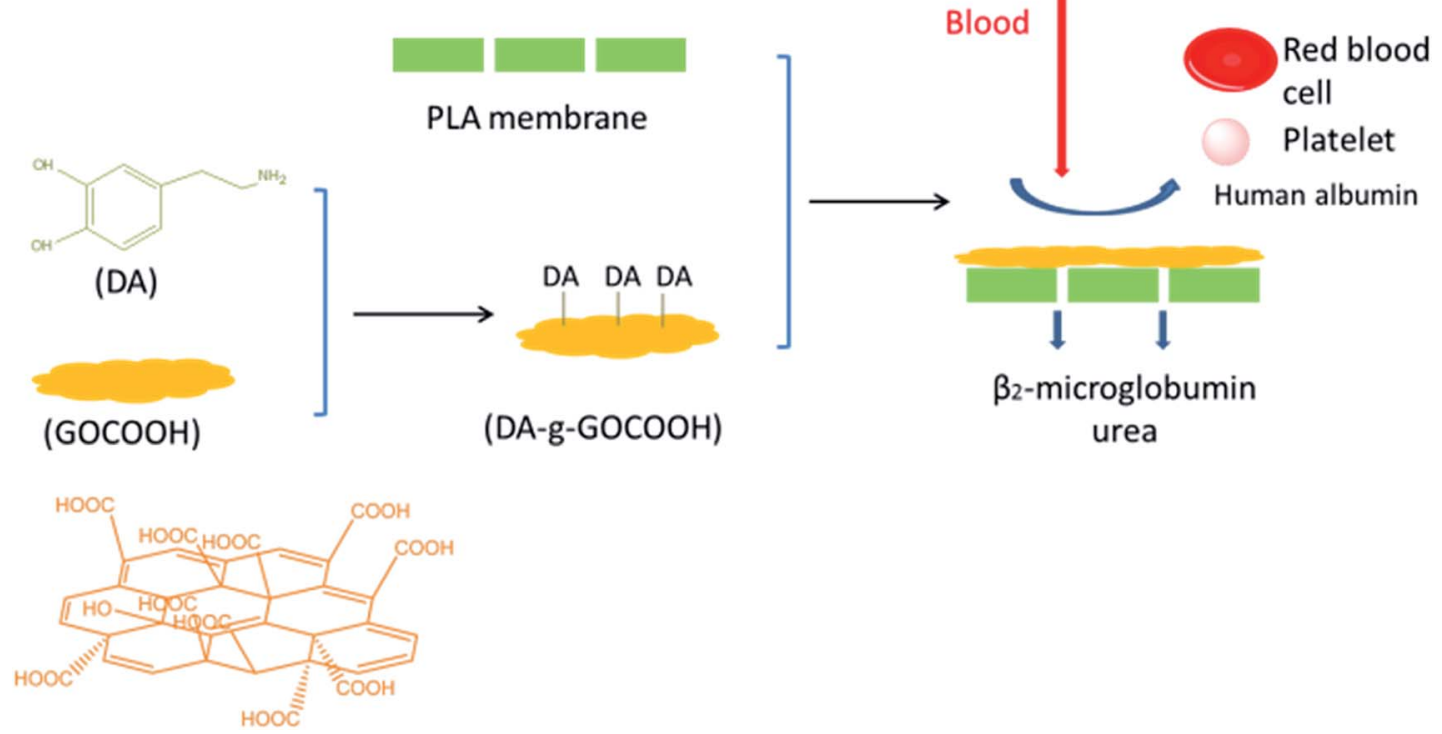

Scheme 1 Schematic procedure of preparing PLA/(DA-g-GOCOOH) membrane, blood anticoagulation and hemodialysis. 
Scanning electron microscopy (SEM) images were acquired by utilizing a scanning electron microscope (SEM, Hitachi S4800, Japan). Attenuated total reflectance Fourier transform infrared spectra (ATR-FTIR, Thermo-Nicolet iS50, America) and X-ray photoelectron spectroscopy (XPS, K-Alpha, ThermoFisher, America) were utilized to analyse the surface chemical composition of the modified PLA membranes. The water contact angle was obtained by using SL200KB (KINO, America). A SurPASS electrokinetic analyser (Anton Paar $\mathrm{GmbH}$, Austria) was utilized to study the zeta potentials of the membranes $\left(1 \mathrm{mmol} \mathrm{L}^{-1} \mathrm{KCl}\right.$ solution, $25^{\circ} \mathrm{C}$ ).

\subsection{Blood compatibility assays}

2.3.1 Platelet adhesion. The anticoagulant sheep whole blood was purchased from Beijing Pingrui Biotechnology Company, China. All the blood experiments were performed in accordance with the guidelines and regulations for the care and use of laboratory animals of the National Institutes of Health. All procedures were approved by the Institutional Animal Care and Use Committee of Tianjin Polytechnic University (Tianjin, China). $5 \mathrm{~mL}$ anticoagulant sheep whole blood was centrifuged at $200 \mathrm{~g}$ for $10 \mathrm{~min}$. The supernatant was platelet-rich plasma (PRP). $100 \mu \mathrm{L}$ of the fresh PRP was added to each well containing one piece of membrane sample $(1 \mathrm{~cm} \times 1 \mathrm{~cm})$ washed with PBS buffer solution ( $\mathrm{pH} 7.4)$ and then maintained at $37^{\circ} \mathrm{C}$ for $1 \mathrm{~h}$. After rinsing in PBS twice gently, the membrane samples were processed with glutaraldehyde $(2.5 \mathrm{wt} \%)$ to fasten the platelets adhesion. Then the samples were gradually dehydrated with changing weight percentage of ethanol/water solutions $(10,30,50,70,90$, and $100 \mathrm{wt} \%)$. SEM was applied to investigate the adhesion of platelets on membranes and the average number of platelets adhered on samples was calculated.

2.3.2 Plasma recalcification time. $5 \mathrm{~mL}$ anticoagulant sheep whole blood was centrifuged at $3000 \mathrm{rpm}$ for $15 \mathrm{~min}$. The platelet-poor plasma (PPP) was obtained by taking out the surpernatant. $100 \mu \mathrm{L}$ PPP was pipetted onto the surface of each sample in a 24-well cell culture plate and incubation $\left(37^{\circ} \mathrm{C}\right)$ was performed for $1 \mathrm{~min}$. Subsequently, $100 \mu \mathrm{L} \mathrm{CaCl}_{2}(0.025 \mathrm{M})$ aqueous solution was added and the addition time was accurately recorded. Stirred the mixture gently with a stainless steel hook to observe the appearance of the first fibrin thread. Then the plasma recalcification time (PRT) was recorded.

2.3.3 Hemolysis ratio (HR). The membranes $(1 \mathrm{~cm} \times 1 \mathrm{~cm})$ were preprocessed in $0.9 \mathrm{wt} \% \mathrm{NaCl}$ at $37^{\circ} \mathrm{C}$ for $40 \mathrm{~min}$. Then, $200 \mu \mathrm{L}$ sheep whole blood was added, incubated at $37^{\circ} \mathrm{C}$ for $1 \mathrm{~h}$. The mixture was centrifuged at $800 \mathrm{~g}$ for $10 \mathrm{~min}$ and the absorbance of the supernatant was obtained by UV/visible spectrophotometer (Ultrospec 2100 pro, GE, America) at $545 \mathrm{~nm}$. $0.9 \mathrm{wt} \% \mathrm{NaCl}$ solution was selected as a negative control and DI water was selected as a positive control. The HR was calculated by the following equation:

$$
\mathrm{HR}=(\mathrm{AS}-\mathrm{AN}) /(\mathrm{AP}-\mathrm{AN}) \times 100 \%
$$

where AS was the absorption value of samples, AN was the absorption value of negative control (saline), and AP was the absorption value of positive control (water).

\subsection{Hemodialysis evaluation tests}

Urea $\left(1.5 \mathrm{~g} \mathrm{~L}^{-1}\right)$, lysozyme $\left(0.04 \mathrm{~g} \mathrm{~L}^{-1}\right)$ and bovine serum albumin (BSA, $\left.1.0 \mathrm{~g} \mathrm{~L}^{-1}\right)$ in physiological saline $(0.9 \mathrm{wt} \%)$ were used as the mimic blood. The flows of the mimic blood and dialysate (pure water) were $100 \mathrm{~mL} \mathrm{~min}^{-1}$ and $300 \mathrm{~mL} \mathrm{~min}^{-1}$, respectively. As the UV adsorption wavelength of BSA was similar to lysozyme, the retention to BSA was measured separately. Each sample was tested for $6 \mathrm{~h}$ (effective membrane area was $7.0 \mathrm{~cm}^{2}$ ).

2.4.1 Water flux. The water flux was measured at $25{ }^{\circ} \mathrm{C}$ under 0.1 MPa. Pure water was filtrated through a membrane sample and the liquid flux was named as $J$, which was calculated by

$$
J=\frac{V}{S \times t}
$$

where $J\left(\mathrm{~L} \mathrm{~m}^{-2} \mathrm{~h}^{-1}\right)$ was the flux, $V(\mathrm{~L})$ was the volume of permeated solution, $S\left(7.0 \mathrm{~cm}^{2}\right)$ was the effective filtration area, $t$ (h) was the operation time.

2.4.2 Urea clearance. $1.5 \mathrm{~mL}$ mimic blood sample was mixed with $0.8 \mathrm{~mL}$ PDAB solution $(0.1 \mathrm{M})$ and $1.7 \mathrm{~mL}$ pure water and incubated at $37{ }^{\circ} \mathrm{C}$ for $10 \mathrm{~min}$. The UV/vis spectrophotometer (Ultrospec 2100 pro, GE, America) (at a wavelength of $410 \mathrm{~nm}$ ) was used to measure the concentration of urea. Then

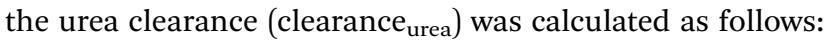

$$
\text { Clearance }_{\text {urea }}(\%)=\left(1-\frac{C_{\text {urea }}^{\text {final }}}{C_{\text {ureainal }}^{\text {orial }}}\right) \times 100 \%
$$

where $C_{\text {urea }}^{\text {original }}$ was the original urea concentration before dial-

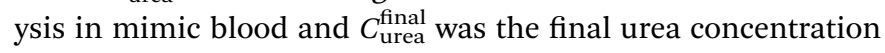
after dialysis in mimic blood.

2.4.3 Lysozyme clearance. A UV/vis spectrophotometer was applied to determine the lysozyme concentration (at a wavelength of $278 \mathrm{~nm}$ ). The lysozyme clearance (clearance lysozyme$_{\text {) }}$ was calculated as follows:

$$
\text { Clearance }_{\text {lysozyme }}(\%)=\left(1-\frac{C_{\text {lysozyme }}^{\text {final }}}{C_{\text {lysozozyme }}^{\text {oriinal }}}\right) \times 100 \%
$$

where $C_{\text {lysozyme }}^{\text {original }}$ was the original lysozyme concentration before dialysis in mimic blood and $C_{\text {lysozyme }}^{\text {final }}$ was the concentration after membrane dialysis in mimic blood.

2.4.4 BSA retention. A UV/vis spectrophotometer was used to obtain the BSA concentration in the mimic blood (at a wavelength of $280 \mathrm{~nm}$ ). The BSA retention (retention ${ }_{\mathrm{BSA}}$ ) was obtained as follows:

$$
\text { Retention }_{\mathrm{BSA}}(\%)=\frac{C_{\mathrm{BSA}}^{\text {final }}}{C_{\mathrm{BSA}}^{\text {corigial }}} \times 100 \%
$$

where $C_{\mathrm{BSA}}^{\text {original }}$ and $C_{\mathrm{BSA}}^{\text {final }}$ were the concentrations in the mimic blood before and after membrane dialysis.

\section{Results and discussion}

\subsection{Preparation and characterization of DA- $g$-GOCOOH nanosheets}

The chemical compositions and morphology of GO-COOH were analysed by FTIR spectroscopy (Fig. S1†) and AFM (Fig. S2 $\dagger$ ). As 
shown in Fig. S1, $\dagger$ the peaks at 1730, 1640 and $1100 \mathrm{~cm}^{-1}$ in the FT-IR spectrum were characteristic of the $\mathrm{C}=\mathrm{O}$ and $\mathrm{C}-\mathrm{O}$ stretches of the ketone groups and epoxy groups, respectively, on GO. Compared with GO, the FT-IR spectrum of GO-COOH showed a stronger - $\mathrm{COOH}$ peak and a bigger peak width, while the $-\mathrm{OH}$ peak became weaker and a smaller peak width. This implied that partial epoxy and hydroxyl were activated into carboxyl. As shown in Fig. S2 $\uparrow$ and 2 the thickness of $\mathrm{GO}-\mathrm{COOH}$ nanosheet was about $1.66 \mathrm{~nm}$ and a 2D surface morphology was observed. The results indicated the GO-COOH nanosheets successfully prepared.

The chemical compositions of the DA- $g$-GOCOOH nanosheets were analysed by FTIR (Fig. 1A) and XPS (Fig. 1B). As shown in Fig. 1A, the peaks at 1730,1630 and $1380 \mathrm{~cm}^{-1}$ appeared in the FTIR spectra of GOCOOH and DA- $g$-GOCOOH were related to the stretching vibration of $\mathrm{C}=\mathrm{O}$ in carboxy group, asymmetric deformation of $\mathrm{COO}^{-}$in carboxy group, and $\mathrm{C}-\mathrm{OH}$ blending vibration in carboxy group respectively. Compared with GOCOOH, a new weak peak at $1280 \mathrm{~cm}^{-1}(\mathrm{C}-\mathrm{N}$ stretching vibrations) appeared in spectra of DA- $g$-GOCOOH.

The peak of $\mathrm{N}-\mathrm{H}$ bending vibrations $\left(1610 \mathrm{~cm}^{-1}\right)$ was overlapped with $\mathrm{COO}^{-}$asymmetric deformation. Therefore, the DA$g$-GOCOOH component was further characterized by XPS. The XPS and the molar percentages of element were shown in Fig. 1B and Table 1. Compared with GOCOOH, the new peak of nitrogen ( $\mathrm{N} \mathrm{1s}$ ) at $400 \mathrm{eV}$ attributed to the $\mathrm{C}-\mathrm{N}$ group could be
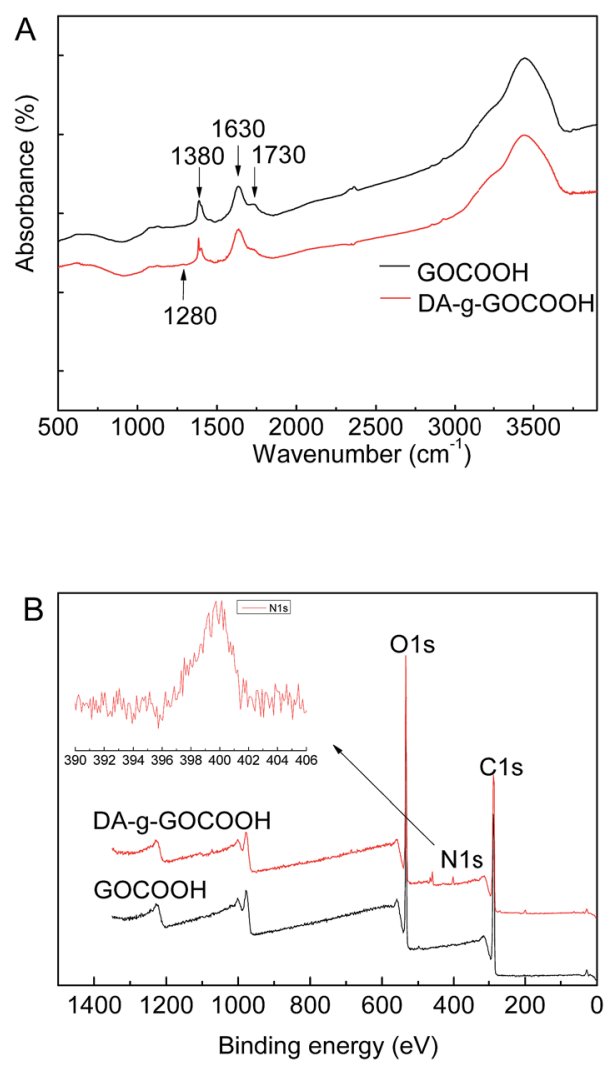

Fig. 1 FTIR spectra (A) and XPS wide scans (B) of the GO and DA-g$\mathrm{GOCOOH}$ nanosheets. The insets in part (B) show the peak of $N 1 \mathrm{~s}$ in detail.
Table 1 The elemental molar percentages determined by XPS

\begin{tabular}{llll}
\hline & \multicolumn{3}{l}{ Percentage of components (mol\%) } \\
\cline { 2 - 4 } Samples & $\mathrm{C}$ & $\mathrm{O}$ & $\mathrm{N}$ \\
\hline GOCOOH & 67.44 & 32.56 & - \\
DA- $g$-GOCOOH & 67.69 & 30.49 & 1.82
\end{tabular}

observed and the molar percentage of $\mathrm{N} 1 \mathrm{~s}$ increased from 0 to $1.82 \%$. Both FTIR and XPS results confirmed that the dopamine molecules were successfully grafted onto GOCOOH nanosheets.

TEM was also employed to detect the morphologies of DA- $g$ GOCOOH nanosheets. As shown in Fig. 2, the wrinkle-like thin sheets, which were the characteristic structure of GO nanosheet, were observed in both GOCOOH and DA- $g$-GOCOOH. The TEM image of DA-g-GOCOOH exhibited a 2D surface morphology. The FTIR spectra, XPS spectra and TEM image
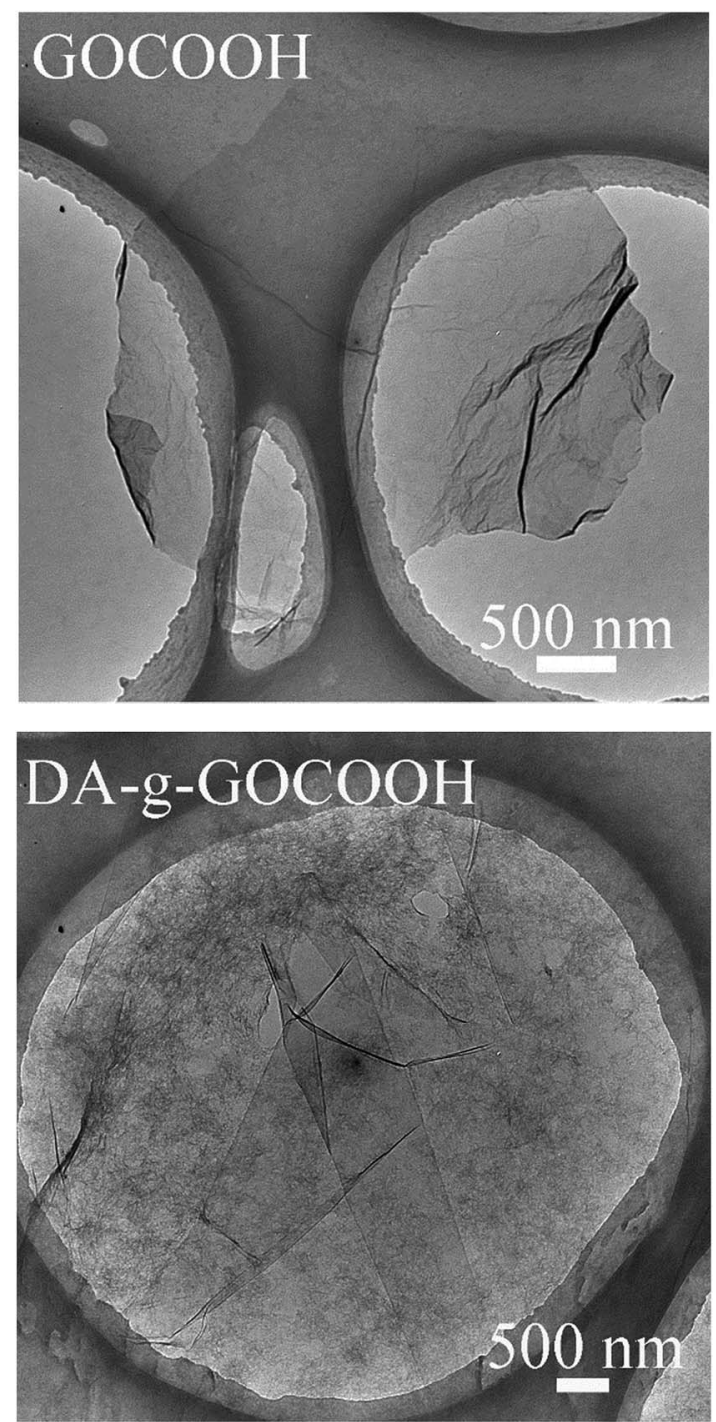

Fig. 2 The TEM images of nanosheets: GOCOOH and DA-g$\mathrm{GOCOOH}$ 
indicated that the DA- $g$-GOCOOH nanosheets were successfully prepared.

DA- $g$-GO nanosheets were shown in Fig. S3. $\dagger$ The molar percentage of $\mathrm{N} 1 \mathrm{~s}$ increased from 0 to $2.02 \%$ (Table S2 $\dagger$ ) and DA- $g$-GO exhibited a 2D surface morphology. The photographs of rGO suspension with different microwave treatment time $(0$, 20, 40, $60 \mathrm{~min}$ ) and XPS wide scans of C 1s in rGO were shown in Fig. S4. $\dagger$

And the $-\mathrm{COOH}$ group contents of DA- $g$-GOCOOH, DA- $g$-GO and rGO were shown in Table S1. $\dagger$ Compared with DA- $g$-GO $\left(2.430 \mathrm{mM} \mathrm{g}^{-1}\right)$, the $-\mathrm{COOH}$ group density was $5.470 \mathrm{mM} \mathrm{g}^{-1}$ in DA-g-GOCOOH. This was related to the carboxylation of GO. However, the $-\mathrm{COOH}$ group density of rGO with $60 \mathrm{~min}$ reduction treatment was $0.310 \mathrm{mM} \mathrm{g}^{-1}$. The $-\mathrm{COOH}$ density had closer relationship with the hydrophilicity and electronegativity of the membranes.

\subsection{Characterization of the modified membranes}

The surface chemical components of the membranes were detected by XPS. The XPS spectra and the molar percentages of element were shown in Fig. 3 and Table 2. There was no nitrogen element in original PLA membrane just as shown in Fig. 3, whereas, the peak of nitrogen (N 1s, molar ratio 3.46\%) at $400 \mathrm{eV}$ attributed to the $\mathrm{C}-\mathrm{N}$ group, was observed on DA- $g$ GOCOOH-immobilized membrane.

The morphologies of membrane surface were observed by SEM. As shown in Fig. 4, the surface of pristine PLA membrane

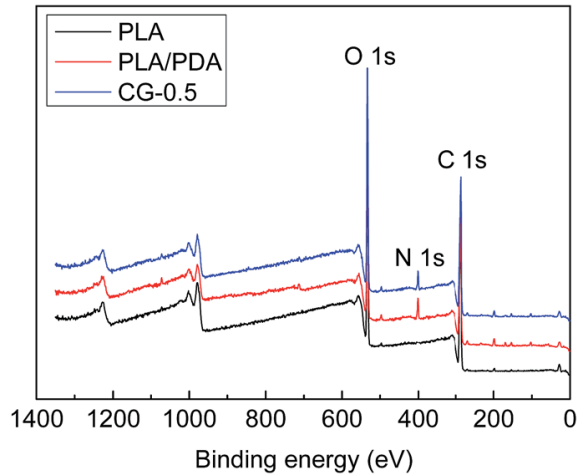

Fig. 3 XPS spectra of PLA, PLA/PDA and CG-0.5 membranes.

Table 2 The elemental molar percentages of PLA, PLA/PDA and PLA$g$-GOCOOH membranes determined by XPS

Percentage of components (mol\%)

\begin{tabular}{llll} 
Samples & $\mathrm{C}$ & $\mathrm{O}$ & $\mathrm{N}$ \\
\hline PLA & 64.67 & 35.33 & - \\
PLA/PDA & 66.35 & 28.82 & 4.83 \\
CG-0.5 & 63.33 & 31.06 & 3.46 \\
CG-1.0 & 63.02 & 31.43 & 3.98 \\
CG-1.5 & 62.59 & 31.73 & 4.18 \\
CG-2.0 & 62 & 29.21 & 4.29
\end{tabular}
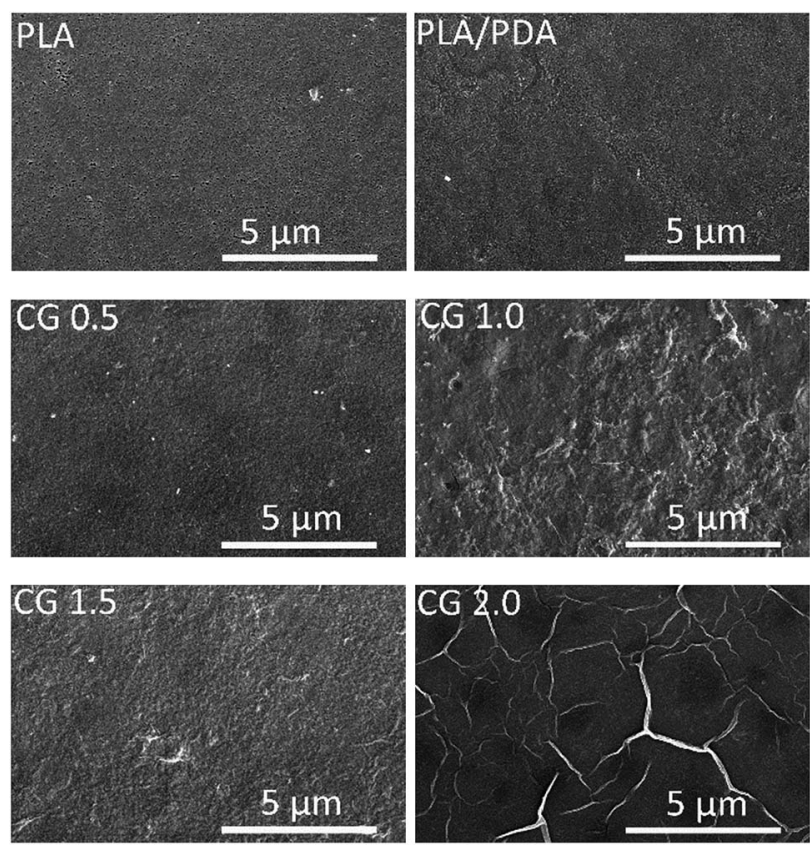

Fig. 4 Surface SEM images of the PLA and DA-g-GOCOOH-coated PLA membranes (CG-0.5, CG-1.0, CG-1.5, CG-2.0, respectively).

was relatively smoother and the pores could be observed. The DA- $g$-GOCOOH adhered onto the PLA membranes surface due to the polymerization of dopamine molecules in alkaline solution. The pore size and pore number of the DA- $g$-GOCOOH modified PLA membranes remarkably decreased. It was related to the immobilizing of DA- $g$-GOCOOH layer. And the wrinklelike structures on membrane surface became more and more apparent with the increasing immersing concentration of the DA- $g$-GOCOOH. The DA- $g$-GOCOOH nanosheets were distinctly observed when the coating concentration of DA- $g$-GOCOOH was $2.0 \mathrm{mg} \mathrm{mL}{ }^{-1}$ (Fig. 4, CG-2.0). The same situation occurred for DA- $g$-GO coated PLA membranes (Fig. S5 $\dagger$ ). The SEM image for rGO coated PLA membranes were shown in Fig. S5. $\uparrow$ The rGO nanosheets with different microwave treatment time could be obviously observed the PLA membranes.

The hydrophilic-hydrophobic properties of membrane surfaces were detected by water contact angle. As shown in Fig. 5A, the contact angle of pure PLA membrane was as high as $77.4^{\circ}$. The contact angle decreased to $66.5^{\circ}$ of the polydopamine coating PLA membrane (PLA/PDA). It was attributed to the hydrophilic groups (-OH and $\left.-\mathrm{NH}_{2}\right)$ in polydopamine. ${ }^{26}$ Compared with the native PLA membrane and the PLA/PDA membrane, the DA- $g$-GOCOOH modified PLA membranes showed remarkably improvement of hydrophilicity, and the water contact angle decreased from $60.3^{\circ}$ to $36.2^{\circ}$ with the increasing immersion concentration of DA- $g$-GOCOOH (from $0.5 \mathrm{mg} \mathrm{mL}^{-1}$ to $2.0 \mathrm{mg} \mathrm{mL}^{-1}$ ). A hydration layer was able to form by the electrostatic interaction and hydrogen bonds. ${ }^{13,32}$ It was related to the abundant carboxy group of DA- $g$-GOCOOH nanosheets.

The membrane surface charge was another key factor to influence the hemocompatibility of the membrane. The zeta 

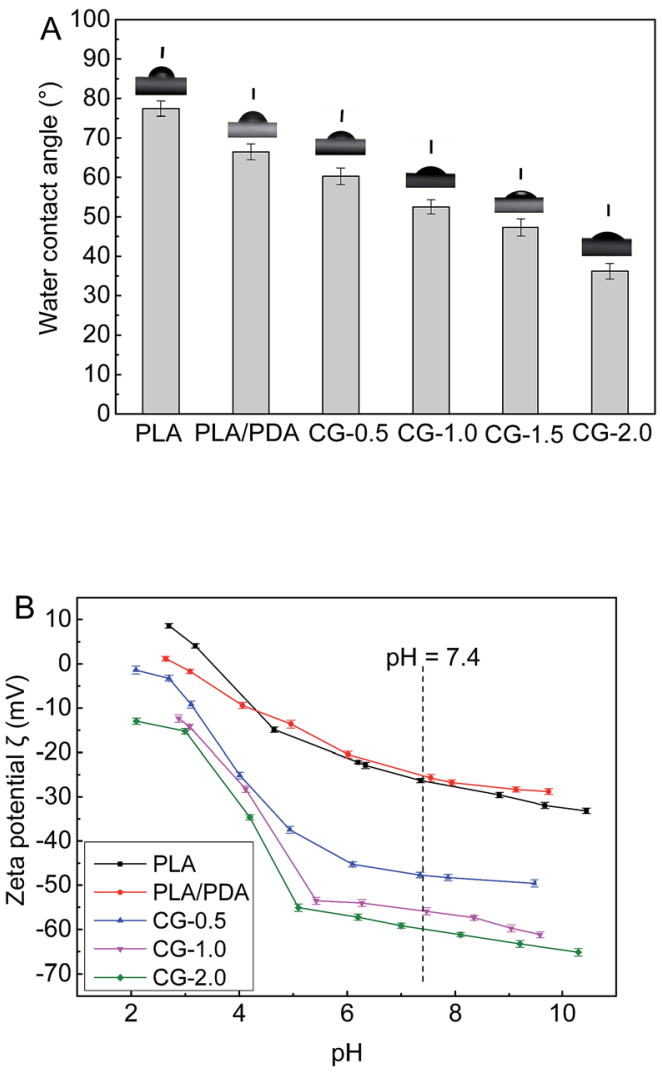

Fig. 5 Water contact angle (A) and zeta potential (B) of membranes. Data were mean $\pm \mathrm{SD}(n=3)$.

potential $(\zeta)$ of the membranes was investigated $(1 \mathrm{mM} \mathrm{KCl}$ solution at $25^{\circ} \mathrm{C}$ ). And as shown in Fig. 5B, the zeta potential of the pure PLA membrane was $-26.6 \mathrm{mV}$ at $\mathrm{pH} 7.4$ (simulating the vivo environment). The PLA/PDA membrane $(-25.0 \mathrm{mV})$ exhibited less electronegative than the native PLA membrane, which might be induced by the amine groups of polydopamine layer. For the DA-g-GOCOOH immobilized PLA membranes (CG-0.5, CG-1.0, CG-2.0), the zeta potential notably decreased from $-47.7 \mathrm{mV}$ to $-59.9 \mathrm{mV}$. However, the rGO and DA- $g$-GO modified PLA membrane exhibited less hydrophilic or electronegative (Fig. S6 $\dagger$ ). It might be related to more - $\mathrm{COOH}$ group at DA-g-GOCOOH. The zeta potential demonstrated that the surfaces of DA- $g$-GOCOOH modified PLA membrane become more negative than that of the original PLA membrane, which was attributed to the more negative groups $(-\mathrm{COOH}$ and $-\mathrm{OH})$ in the immobilized layer of DA- $g$-GOCOOH. The adsorption of negative platelets was restrained by the electrostatic repulsion for the DA-g-GOCOOH modified PLA membranes. In brief, both the surface hydration and electrostatic was able to benefit the hemocompatibility of PLA membranes by immobilizing DA-gGOCOOH.

\subsection{Hemocompatibility}

Blood coagulation was a vital factor in hemocompatibility. In the present work, the anticoagulation properties of the original PLA membrane and the DA- $g$-GOCOOH modified PLA membranes were evaluated by the platelet adhesion and plasma recalcification time.

Platelets adhesion on the original and modified PLA membranes was investigated by SEM. And the results were shown in Fig. 6. The average number of adhered platelets on each sample was calculated based on SEM images. It was found that remarkable aggregation of the platelets on the native PLA and polydopamine coated membranes occurred. Besides, the platelets spread in irregular shapes on the surface of the PLA and PLA/PDA membranes were observed. This indicated that the platelets were deformed and activated. More platelets adhered on the PLA/PDA membrane surface (about $2.789 \times 10^{6}$ cell per $\mathrm{cm}^{2}$ ) than that of the native PLA membrane (about 2.324 $\times 10^{6}$ cell per $\mathrm{cm}^{2}$ ). It might be induced by the less surface charge of PLA/PDA membrane than the original PLA membrane. For the DA-g-GOCOOH immobilized PLA membranes, the number of adhered platelets notably decreased from $1.046 \times 10^{6}$ cell per $\mathrm{cm}^{2}$ (CG-0.5) to $0.116 \times 10^{6}$ cell per $\mathrm{cm}^{2}$ (CG-2.0). Rarely platelets were observed and the platelets expressed with nearly no deformation on CG-2.0 membrane as shown in Fig. 6. It indicated that immobilizing DA-g-GOCOOH nanosheets on PLA membranes was able to obviously suppress the activation and transmutation of platelets. The hemocompatibility of PLA membranes efficiently improved with the hydrophilic and electronegative membrane surfaces modified by DA-g-GOCOOH.

The blood-clotting of PLA membranes before and after modification was investigated by the plasma recalcification time (PRT). As shown in Fig. 7A, the PRT of the DA-g-GOCOOH immobilized PLA membranes was prolonged to $220 \mathrm{~s}$ (Fig. 7, CG-2.0) comparing with original PLA membrane (120 s). The increased PRT indicated that the plasma coagulation on DA- $g$ GOCOOH immobilized PLA membranes was restrained, which was related to the improvement of the hydrophilicity and electronegativity of the DA- $g$-GOCOOH modified membranes.

Hemolysis ratio (HR) was used to investigate the damage caused by materials on erythrocyte. And the results were shown in Fig. 7B. It was found that the HR value of the original PLA and PLA/PDA membrane was $10.7 \%$ and $5.9 \%$, respectively, which were out of safety level for biomaterials. Compared with DA-gGOCOOH modified membranes, the PLA/PDA membrane is less hydrophilic and electronegative, leading a higher hemolysis ration. The HR value of DA- $g$-GOCOOH immobilized membranes notably reduced to less than $0.3 \%$ (CG-0.5: 0.23\%; CG-1.0: $0.2 \%$; CG-2.0: $0.13 \%$, respectively). This was related to the enhancement of hydrophilicity and electronegativity of the modified membranes. All results indicated that coating DA- $g$ GOCOOH on PLA membrane surface not only prevented platelets adhesion and blood coagulation but also weakened the damage to erythrocyte.

The SEM images of platelets adhesion, recalcification time and hemolysis ratio data of $\mathrm{rGO}$ and DA- $g$-GO modified membrane surfaces were shown in Fig. S7 and S8. $\dagger$ More platelets adhered on the surface, shorter recalcification time and higher hemolysis ratio of rGO and DA- $g$-GO modified PLA membranes than those of DA- $g$-GOCOOH modified PLA membranes. It was attributed to the less hydrophilicity and 

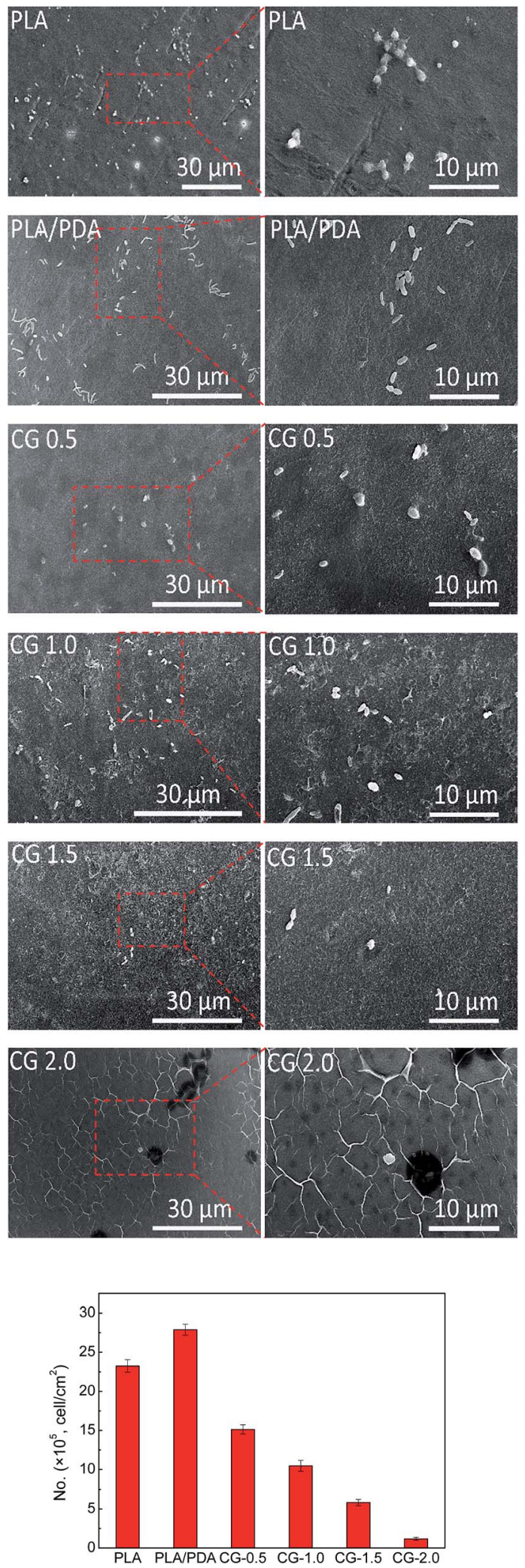

Fig. 6 The representative SEM images of platelets adhered on membrane surfaces: original PLA membrane; PLA/PDA membrane; (CG 0.5, CG 1.0, CG 1.5, CG 2.0) PLA/(DA-g-GOCOOH) membranes with $\mathrm{DA}-\mathrm{g}-\mathrm{GOCOOH}$ immersing concentration of $0.5,1.0,1.5$ and $2.0 \mathrm{mg} \mathrm{mL}^{-1}$, respectively. The number of the adhered platelets on the membrane surfaces. Data were mean $\pm \mathrm{SD}(n=3)$.
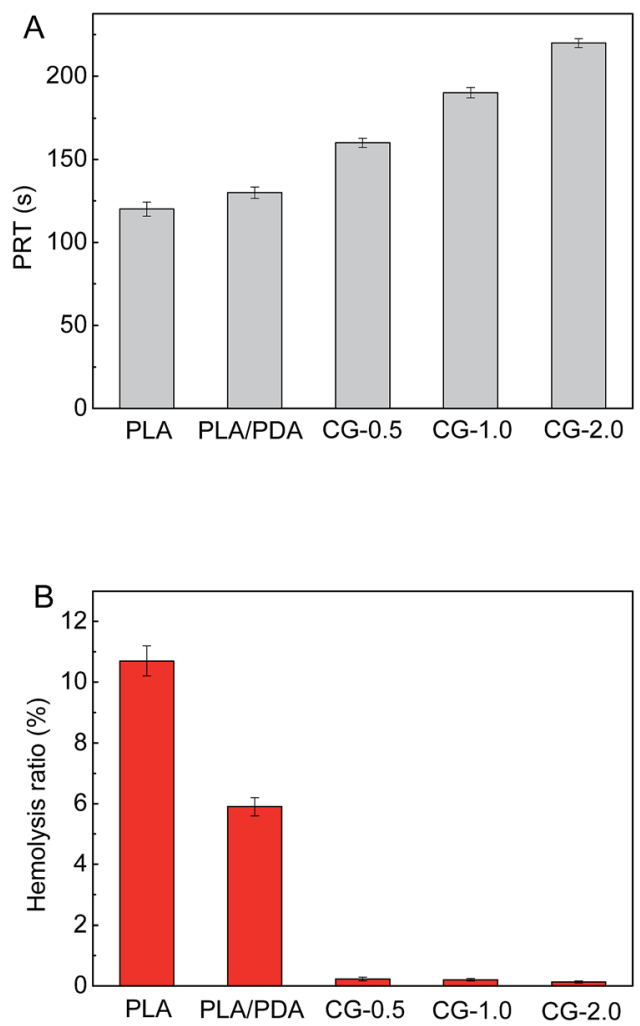

Fig. 7 (A) The plasma recalcification time (PRT) and (B) hemolysis ratio $(\mathrm{HR})$ for the PLA membranes before and after surface modification. Data were mean \pm SD $(n=3)$.
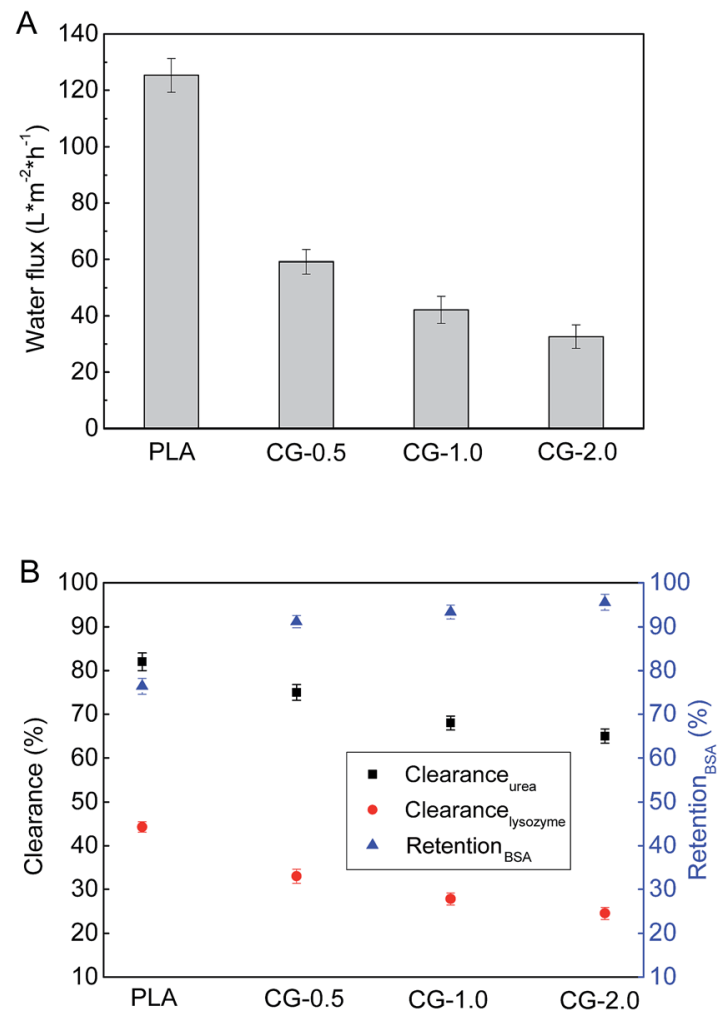

Fig. 8 (A) Pure water flux and (B) urea clearance (clearance urea $_{\text {), lysozyme }}$ clearance (clearance lysozyme $_{\text {) }}$ and BSA retention (retention BSA $_{\text {) }}$ of the PLA and PLA/(DA-g-GOCOOH) membrane. Data were mean \pm SD $(n=3)$. 
Table 3 The performance of hemocompatibility and dialysis in this work compared with references

\begin{tabular}{|c|c|c|c|c|c|c|c|}
\hline Samples & $\begin{array}{l}\text { Platelets adhesion } \\
\text { number }\left(10^{5} \text {, cell per } \mathrm{cm}^{2}\right)\end{array}$ & $\begin{array}{l}\text { Recalcification } \\
\text { time }(\mathrm{s})\end{array}$ & $\begin{array}{l}\text { Hemolysis } \\
\text { rate }(\%)\end{array}$ & $\begin{array}{l}\text { BSA retention } \\
(\%)\end{array}$ & $\begin{array}{l}\text { Lysozyme } \\
\text { clearance (\%) }\end{array}$ & $\begin{array}{l}\text { Urea } \\
\text { clearance (\%) }\end{array}$ & References \\
\hline HEP05-M & - & 270 & 1.36 & 90.8 & 17.9 & 79 & 7 \\
\hline DA-Hep/PES $(24 \mathrm{~h})$ & 2.2 & 70 & - & - & - & - & 12 \\
\hline HSCS-H1 & 34 & 95 & 0.5 & 95.9 & - & 100 & 34 \\
\hline CG 2.0 & 1.16 & 220 & 0.13 & 95.6 & 24.5 & 65 & Present work \\
\hline
\end{tabular}

electronegativity than that of DA-g-GOCOOH modified membrane.

The stability of the DA-g-GOCOOH coating layers was also investigated. The UV-vis absorbance of DA-g-GOCOOH for PLA and modified membranes after incubating different time in water was shown in Fig. S9† and the platelet adhesion on the original PLA and modified PLA membranes after incubating $48 \mathrm{~h}$ in water was shown in Fig. S10. $\dagger$ As shown in Fig. S9, $\dagger$ there was no obvious absorbance peak at $235 \mathrm{~nm}$ (ref. 17) (GO-COOH) or $420 \mathrm{~nm}$ (ref. 33) (polydopamine) after incubating the PLA/ (DA-g-GOCOOH) membrane (CG-2.0) in water for $48 \mathrm{~h}$. However, two peaks at $240 \mathrm{~nm}$ and $278 \mathrm{~nm}$ appeared after incubating 4 days for the blank controls and the PLA/(DA- $g$-GOCOOH) membrane. This phenomenon might be attributed to deterioration of the water. As shown in Fig. S10, $\dagger$ the PLA/(DA- $g$ $\mathrm{GOCOOH}$ ) membranes still restrained the platelet adhesion on membrane surface compared with the original PLA membrane after incubating in water for $48 \mathrm{~h}$. It indicated that the DA-gGOCOOH coating layers were relatively stable.

\subsection{Dialysis performance}

The pure water fluxes of PLA and modified membranes were evaluated, and the results were shown in Fig. 8A. The water flux of the original PLA membrane was $125.3 \mathrm{~L} \mathrm{~m}^{-2} \mathrm{~h}^{-1}$, and the water flux of modified membranes decreased from 59.2 to $32.6 \mathrm{~L}$ $\mathrm{m}^{-2} \mathrm{~h}^{-1}$ with the increment of DA-g-GOCOOH immersing concentration. It indicated that the immobilized DA- $g$ GOCOOH layer was able to decrease the pore size of the membranes leading to the increment of water permeation resistance.

The urea clearance (clearance $_{\text {urea }}$ ), lysozyme clearance (clearance $_{\text {lysozyme }}$ ) and BSA retention (retention ${ }_{\text {BSA }}$ ) of the membranes were utilized to evaluate the hemodialysis performances of the membranes. As shown in Fig. 8B, for the pure PLA membranes, clearance ${ }_{\text {urea }}$, clearance lysozyme and retention ${ }_{\mathrm{BSA}}$ were $82 \%, 44.3 \%$ and $76.4 \%$, respectively. And clearance $_{\text {urea }}$ and clearance lysozyme $_{\text {of the PLA/(DA-g-GOCOOH) }}$ membranes decreased with the increment of DA- $g$-GOCOOH immersing concentration, while the retention ${ }_{\mathrm{BSA}}$ increased. The clearance $_{\text {urea }}$ of the DA- $g$-GOCOOH immobilized membranes decreased from $75 \%$ to $65 \%$, and the clearance lysozyme $_{\text {decreased }}$ from $33 \%$ to $24.5 \%$, while the retention ${ }_{\mathrm{BSA}}$ increased from $91.2 \%$ to $95.6 \%$ when immersing concentration of $\mathrm{DA}-g$ -
GOCOOH increased from $0.5 \mathrm{mg} \mathrm{mL} \mathrm{m}^{-1}$ to $2.0 \mathrm{mg} \mathrm{mL}^{-1}$. Both the pore size and the charge of the membrane surface affected the dialysis performances. The molecular weight of urea, lysozyme and BSA is $60.06 \mathrm{Da}, 14 \mathrm{kDa}$ and $66.4 \mathrm{kDa}$, respectively. The pore size of DA- $g$-GOCOOH coated PLA membranes decreased significantly comparing with that of the pure PLA membrane, and thus the clearance of urea and lysozyme decreased while the retention of BSA increased. Besides, the electronegativity of the modified PLA membranes increased with the DA-g-GOCOOH immersing concentration (see Fig. 6) and therefore the retention of BSA increased. In brief, the dialysis performances of the DA- $g$-GOCOOH modified PLA membranes with negative charge were influenced by the pore size and space charge effect. The performance of hemocompatibility and dialysis in this work compared with references was shown in Table 3.

\section{Conclusions}

The fabrication of hemocompatible PLA hemodialysis membranes by a facile mussel-inspired method was developed in this work. A stable DA-g-GOCOOH layer was formed via immobilizing DA-g-GOCOOH nanosheets onto PLA membranes. The obtained PLA/(DA- $g$-GOCOOH) membranes exhibited good hydrophilicity and enhanced electronegativity. Moreover, the hemocompatibility of the modified PLA membranes was improved involving less platelets adhesion, longer plasma recalcification time and lower hemolysis ratio. In addition, the DA- $g$-GOCOOH immobilized PLA membrane cleared almost $65 \%$ urea, $24.5 \%$ lysozyme and retained $95.6 \%$ BSA. Immobilizing DA- $g$-GOCOOH on the surface of membranes provided a promising avenue to improve the hemocompatibility of hemodialysis membranes.

\section{Conflicts of interest}

There are no conflicts to declare.

\section{Acknowledgements}

The authors gratefully acknowledge the financial support from the National Natural Science Foundation of China (Funding No. 51373120, 51503146), the Science and Technology Commission Foundation of Tianjin (No. 15JCZDJC37600), the Program for 
Changjiang Scholars and Innovative Research Team in University (PCSIRT) of Ministry of Education of China (Grand No. IRT13084) and the Science and Technology Plans of Tianjin (No. 15PTSYJC00240, 15PTSYJC00230).

\section{References}

1 R. M. Rasal, A. V. Janaorkar and D. E. Hirt, Prog. Polym. Sci., 2010, 35, 338-356.

2 L.-J. Zhu, F. Liu, X.-M. Yu, A.-L. Gao and L.-X. Xue, J. Membr. Sci., 2015, 475, 469-479.

3 G. S. Nyanhongo, R. D. Rodríguez, E. N. Prasetyo, C. Caparrós, C. Ribeiro, V. Sencadas, S. Lanceros-Mendez, E. H. Acero and G. M. Guebitz, React. Funct. Polym., 2013, 73, 1399-1404.

4 C. Li, B. Cai, J. Jin, J. Liu, X. Xu, J. Yin and L. Yin, J. Mater. Chem. B, 2015, 3, 8410-8420.

5 J.-H. Jiang, L.-P. Zhu, X.-L. Li, Y.-Y. Xu and B.-K. Zhu, J. Membr. Sci., 2010, 364, 194-202.

6 I. You, S. M. Kang, Y. Byun and H. Lee, Bioconjugate Chem., 2011, 22, 1264-1269.

7 A.-L. Gao, F. Liu and L.-X. Xue, J. Membr. Sci., 2014, 452, 390399.

8 Y. Chang, W. J. Chang, Y. J. Shih, T. C. Wei and G. H. Hsiue, ACS Appl. Mater. Interfaces, 2011, 3, 1228-1237.

9 S. H. Chen, Y. Chang, K. R. Lee, T. C. Wei, A. Higuchi, F. M. Ho, C. C. Tsou, H. T. Ho and J. Y. Lai, Langmuir, 2012, 28, 17733-17742.

10 Z. Xiong, F. Liu, H. Lin, J. Li and Y. Wang, ACS Biomater. Sci. Eng., 2016, 2, 2207-2216.

11 J. Li, F. Liu, Y. Qin, J. He, Z. Xiong, G. Deng and Q. Li, J. Membr. Sci., 2017, 523, 505-514.

12 L. Ma, H. Qin, C. Cheng, Y. Xia, C. He, C. Nie, L. Wang and C. Zhao, J. Mater. Chem. B, 2014, 2, 363-375.

13 D. R. Dreyer, S. Park, C. W. Bielawski and R. S. Ruoff, Chem. Soc. Rev., 2010, 39, 228-240.

14 S. A. Sydlik, S. Jhunjhunwala, M. J. Webber, D. G. Anderson and R. Langer, ACS Nano, 2015, 9, 3866-3874.

15 Y. Chong, Y. Ma, H. Shen, X. Tu, X. Zhou, J. Xu, J. Dai, S. Fan and Z. Zhang, Biomaterials, 2014, 35, 5041-5048.
16 A. B. Seabra, A. J. Paula, R. d. Lima, O. L. Alves and N. Durán, Chem. Res. Toxicol., 2014, 27, 159-168.

17 Z. Ding, Z. Zhang, H. Ma and Y. Chen, ACS Appl. Mater. Interfaces, 2014, 6, 19797-19807.

18 C. Cheng, S. Li, S. Nie, W. Zhao, H. Yang, S. Sun and C. Zhao, Biomacromolecules, 2012, 13, 4236-4246.

19 M. Hu and B. Mi, Environ. Sci. Technol., 2013, 47, 3715-3723.

20 H.-R. Chae, J. Lee, C.-H. Lee, I.-C. Kim and P.-K. Park, J. Membr. Sci., 2015, 483, 128-135.

21 Y.-H. So, H.-T. Chang, W.-J. Chiu and C.-C. Huang, Biomater. Sci., 2014, 2, 1332-1337.

22 C. He, Z. Q. Shi, C. Cheng, H. Q. Lu, M. Zhou, S. D. Sun and C. S. Zhao, Biomater. Sci., 2016, 4, 1431-1440.

23 A. M. Pinto, S. Moreira, I. C. Gonçalves, F. M. Gama, A. M. Mendes and F. D. Magalhães, Colloids Surf., B, 2013, 104, 229-238.

24 T. T. Rahila Batul, A. Khaliqb and A. Yu, Biomater. Sci., 2017, 5, 1204-1229.

25 H. Lee, S. M. Dellatore, W. M. Miller and P. B. Messersmith, Science, 2007, 318, 426-430.

26 Y. Liu, K. Ai and L. Lu, Chem. Rev., 2014, 114, 5057-5115.

27 J. Jiang, L. Zhu, L. Zhu, B. Zhu and Y. Xu, Langmuir, 2011, 27, 14180-14187.

28 H.-C. Yang, J. Luo, Y. Lv, P. Shen and Z.-K. Xu, J. Membr. Sci., 2015, 483, 42-59.

29 Y. Wang, J. Wang, M. Gao and X. Zhang, J. Mater. Chem. B, 2015, 3, 8711-8716.

30 L. Ma, C. Cheng, C. Nie, C. He, J. Deng, L. Wang, Y. Xia and C. Zhao, J. Mater. Chem. B, 2016, 4, 3203-3215.

31 C. Nie, C. Cheng, Z. Peng, L. Ma, C. He, Y. Xia and C. Zhao, J. Mater. Chem. B, 2016, 4, 2749-2756.

32 J. A. Prince, S. Bhuvana, V. Anbharasi, N. Ayyanar, K. V. K. Boodhoo and G. Singh, J. Membr. Sci., 2016, 500, 76-85.

33 C. Zhang, Y. Ou, W.-X. Lei, L.-S. Wan, J. Ji and Z.-K. Xu, Angew. Chem., Int. Ed. Engl., 2015, 55, 3054-3057.

34 R. Wang, Y. Xie, T. Xiang, S.-D. Sun and C.-S. Zhao, J. Mater. Chem. B, 2017, 5, 3035-3046. 